\title{
Peripheral Osteoma of Mandible
}

\author{
Dr. Gururaju C.R ${ }^{1}$, Dr. Usha Chikkaiah ${ }^{2}$ \\ 1. Reader, Department of Oral \& Maxillofacial Surgery, Sharavathi Dental College and Hospital, Shivamogga- \\ 577204, Karnataka, India \\ 2. Reader, Department of Oral Medicine \& Radiology, Sharavathi Dental College and Hospital, Shivamogga- \\ 577204, Karnataka, India
}

\begin{abstract}
Most of the head and neck tumors are odontogenic in origin. Non odontogenic tumors of epithelial or mesenchymal origin also form a significant component in this region. The tumors arising from mesenchymal component may originate from various structures like fibrous tissue, adipose tissue, bone and cartilage. Here we report a case of benign tumor originating from bone that is restricted to the craniofacial skeleton.
\end{abstract}

Key words: Tumors, Benign Bone tumor, Craniofacial skeleton.

\section{Introduction:}

In jaws where infection is common, it is not always possible to differentiate a bony mass induced by irritation or inflammation from a true neoplastic origin as in other parts of the body. ${ }^{1}$ In addition the developmental disturbances of jaws like exostoses and endostoses further complicate the diagnosis, as they may produce most similar clinical, radiographic and histologic picture. ${ }^{1}$ Osteoma is one such benign bone tumor that is restricted to the craniofacial skeleton rarely affecting the extragnathic skeleton. ${ }^{2}$ Osteoma is considered as an inactive neoplasm, most often an accidental finding on radiographic examination. ${ }^{3,4}$ The symptoms like sinusitis, localized pain, headache, nasal obstruction, exopthalmosis, facial asymmetry and difficulty in opening the mouth is attributed depending on the site of occurance. ${ }^{1,3,4}$ In the present case facial asymmetry was of main concern.

\section{Case Report:}

A 23 years old male patient reported with swelling in left body of mandible. The lesion was totally asymptomatic. No functional restrictions other than slight facial asymmetry. History of slow growing since 6 months. On extraoral examination, slight facial asymmetry was noted on left parasymphyseal region on buccal aspect of the mandible. Swelling measured approximately $1 \mathrm{X} 1.5 \mathrm{~cm}$, which was nontender, firm to hard in consistency on palpation and was immobile (figure 1). The skin over the swelling did not show any secondary changes. Cervical lymph nodes were not palpable. Intra orally no abnormalities were detected in both soft and hard tissue examination. Occlusal radiograph showed a well circumscribed radio opaque mass in relation to the left buccal cortical plate at parasymphyseal region measuring about $1.5 \mathrm{X} 1 \mathrm{~cm}$ in size (Figure 2). A provisional diagnosis of osteoma was considered. Excision of the lesion was done through intra oral approach under local anaesthesia (Figure 3). The gross specimen measuring about 1 X $1.5 \mathrm{~cm}$ was sent to histopathological examination. Histologically it was well encapsulated with trabeculae of bone arranged in lace like pattern having osteoblastic rimming and osteocytes within the lacunae with evidence of resting lines at centre and at the periphery it was lined by plump osteoblasts suggestive of cancellous osteoma (Figure 4).

\section{Discussion:}

Osteoma was described as a specific entity by Jaffer in 1935 and since then hundreds of cases have been published which bear out his original criteria stating: the lesion being a benign neoplasm which formed large amounts of osteoid later getting calcified with a little evidence to suggest that the lesion was an inflammatory process, and with radiographic changes such as focal rarefaction and reactive bone, which appeared some distance from the lesion, which frequently occurring in young adults and pain is an outstanding feature (not so anymore) and complete removal is the treatment of choice. ${ }^{1,4}$ Shafer et al in 1983 described osteomas as a benign neoplasm characterized by proliferation of compact or cancellous bone, usually in an endosteal or periosteal location and rarely entirely in soft tissues. ${ }^{1,5}$ The central osteomas arises in medullary (endosteal) bone and peripheral ostomas arises on the bone surface as a polypoid or sessile mass from periosteum. ${ }^{3,5,6}$ The extraskeletal soft tissue osteoma usually developing within the muscle. ${ }^{6}$

\subsection{Etiopathogenesis:}

The exact etiology and pathogenesis of peripheral osteoma is unknown. Both hamartomatous and neoplastic factors have been advocated with no definite conclusion. Infiltration of interdental bone and abnormal 
histological bone structure might suggest the neoplastic nature. ${ }^{5,6}$ Various others have attributed developmental, neoplastic and reactive causes as possible etiologic factors. It is unlikely that peripheral osteomas are developmental anomaly as most cases occur in adults. These are also probably not neoplastic because of their slow growth rate. Some investigators have classified them as a reactive condition triggered by trauma, as they are generally located on the lower border or buccal aspect of the mandible which are susceptible to trauma. As many are located in close proximity to muscle attachment (masseter, median pterygoid, temporalis) it is possible that muscle traction may play a role in development of peripheral osteomas. ${ }^{6}$ The suggested mechanism that best explains its pathogenesis is a combination of trauma and muscle traction. The former may cause subperiosteal bleeding and odema, latter could be related to local elevation of the periosteum. These two probabilities might initiate an osteogenic reaction that could be perpetuated by the continous muscle traction in the area. ${ }^{4,5,6}$

\subsection{Clinical Features:}

Osteomas can occur at any age, found most frequently in young adults. Osteomas in maxillofacial region have been reported in patients between 29.4 and 40.5 years. It usually remains less than two $\mathrm{cm}$ in size after years of slow enlargement. ${ }^{1,2}$ They are more frequent in males than in females approximately $2: 1{ }^{2,5}$ The extragnatic osteomas are more commonly found in the cortical plate of long bones such as the femur and the tibia. ${ }^{4}$ In the maxillofacial region, osteomas occur most frequently in the sinuses. The most common site is the frontal sinus, followed by ethmoidal and maxillary sinuses. ${ }^{2}$ Other locations include the external auditory canal, orbit, temporal bones and pterygoid plates. ${ }^{3}$ With regard to the facial bones, it is more common in the mandible than in the maxilla and lingual surface of the mandibular body posterior to premolars and lower border in the angle region. ${ }^{5}$ Most of the osteomas occurring in the mandible are dense osteomas and the cancellous osteoma is comparatively rare. ${ }^{4}$ In present case the lesion occurred in buccal parasymphyseal region and histologic picture was suggestive of cancellous osteoma which is a rare entity.

Peripheral osteomas are normally an incidental finding since they are asymptomatic, but other times depending on the location and the size of the lesion, it can cause facial deformity, deviation of the mandible on opening the mouth, headache, bone pain, dysphagia or exopthalmosis. The most common symptom when present is pain. ${ }^{5,6}$

\subsection{Radiographic \& Histologic features:}

Conventional radiological examinations are generally sufficient to diagnose an osteoma. Radiographically it appears as a unilateral, pedunculated, well defined, oval or round mushroom like radiopaque mass with similar density to normal bone. ${ }^{2,3,5}$ Histologically, osteoma consists of mature, lamellar bone with minimal marrow tissue (compact osteoma) or of trabeculae of mature lamellar bone with intervening fatty or fibrous marrow (cancellous osteoma). ${ }^{3,5}$

\subsection{Differential diagnosis:}

The various differential diagnosis considered for osteomas are osteochondroma, fibrous dysplasia, chondroma, ossifying fibroma, condensing osteitis, exostoses including tori, which are bony excrescences that occur on the buccal aspect of the alveolar bone. Bony exostoses (except tori) tend to appear on the buccal/facial aspects of the alveolar bone in contrast to peripheral osteomas, which almost always occur on the lingual/palatal aspect. Focal sclerosing osteomyelitis, osteosarcoma, peripheral ossifying fibroma, chondroma, Pagets disease, monostotic fibrous dysplasia, calcified meningioma and odontoma should also be considered in the differential diagnosis. These lesions are of reactive or developmental origin and are not thought to be true neoplasms. Osteoblastomas and osteoid osteoma are usually more painful and have a greater growth rate than osteomas. ${ }^{3,4,5,6}$

The presence of osteomas may be a sign of the presence of Gardners syndrome, which is characterized by a triad of colorectal polyposis, skeletal abnormalities (with multiple osteomas of the skull and face) and multiple impacted supernumerary teeth. Since the osteoma develop before the colorectal polyposis, early recognition of the syndrome is very important to the prognosis of the disease. ${ }^{2,5}$

\subsection{Treatment \& Prognosis:}

There are no reports of malignant transformation of peripheral osteoma. Asymptomatic lesions are left untreated and regular follow up is done. Surgery is indicated when there are symptoms, deformity or if the lesion presents active growth. Recurrence is extremely rare. ${ }^{2,3}$

\section{Conclusion:}

In conclusion, the osteomas are slow growing benign tumors with a very rare recurrence rate. The identification of the radiographic features is essential to diagnose the osteoma, since its radiographic appearance is characteristic and should be kept in mind in order to the diagnosis. Although its etiology remains unknown we 
could suggest the traumatic cause for the present case. Periodical clinical and radiographic follow ups are to be performed for osteomas after surgical removal.

\section{Conflict Of Interest: Nil}

\section{Source Of Funding: Nil}

\section{Acknowledgement: Nil}

\section{References:}

[1]. Shafer, Hine, Levy. Shafers text book of Oral Pathology. $5^{\text {th }}$ edn benign and malignant tumors of oral cavity pg 212-15

[2]. Anna Reis Duido et al Osteoma of the Zygomatic arch and mandible; report of two cases. Rev Port estimated Med Dent Cis Maxillofac 2012;53(2):103-7

[3]. Alejendro Donohue, Cornejo et al. Active giant peripheral osteoma of the mandible, presentation of one case with follow up to 6yrs. J Clin Exp Dent 2010;294):e212-4

[4]. Mayur chaudhary, Meena Kulkarni. Osteoid osteoma of mandible. JOMFP 2007; 11(2): 52-55

[5]. Etienne Romanelli Jerra et al. Peripheral osteoma of the mandible: clinical case. Braz J Oral Sci April/June 2005;4(13/

[6]. Lt.Col IO Roy. Peripheral osteoma of Mandible. MJAFI 2008; 64:385-86

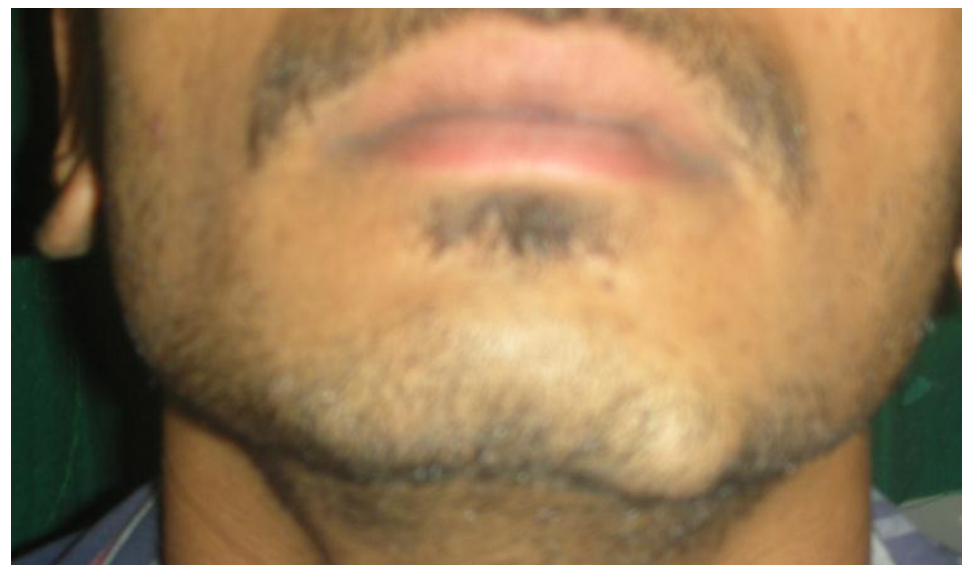

Figure 1: Extra oral view, the patient presents a hard and visible mass at the left mandibular parasymphyseal region

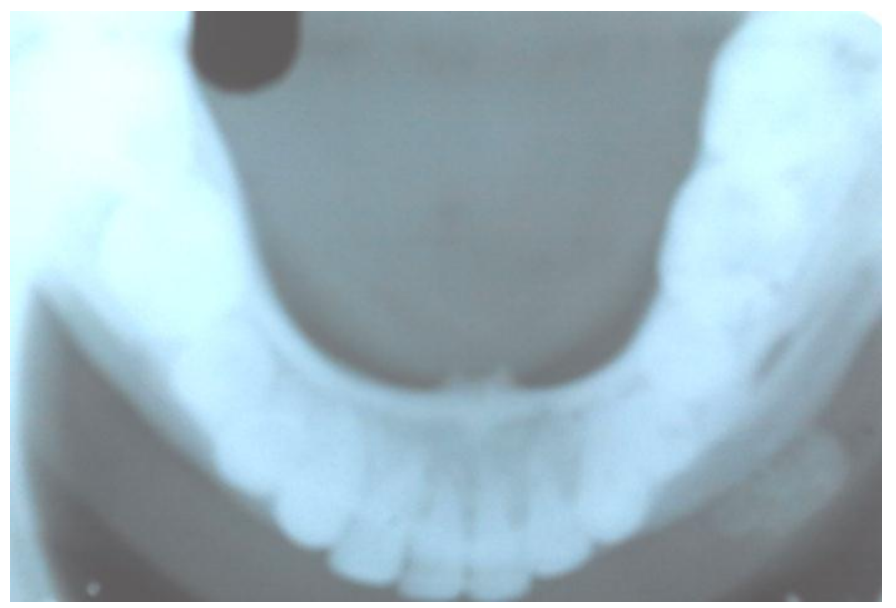

Figure 2: Occlusal radiograph showing radio opaque lesion at left buccal parasymphyseal region 


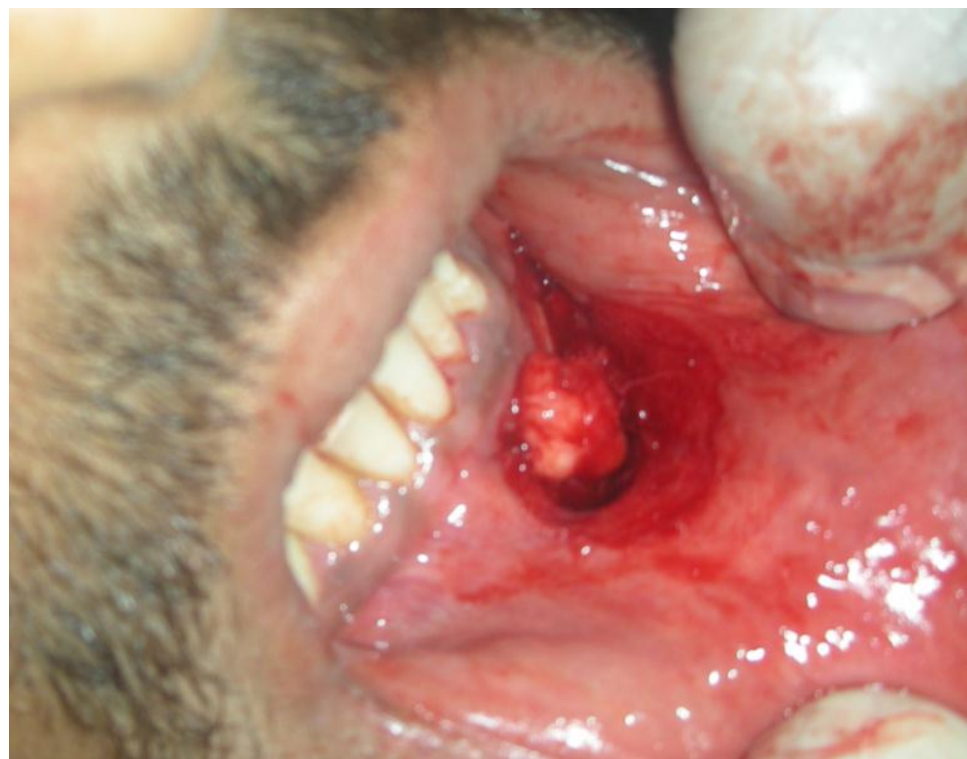

Figure 3: Intra operative view of surgical site

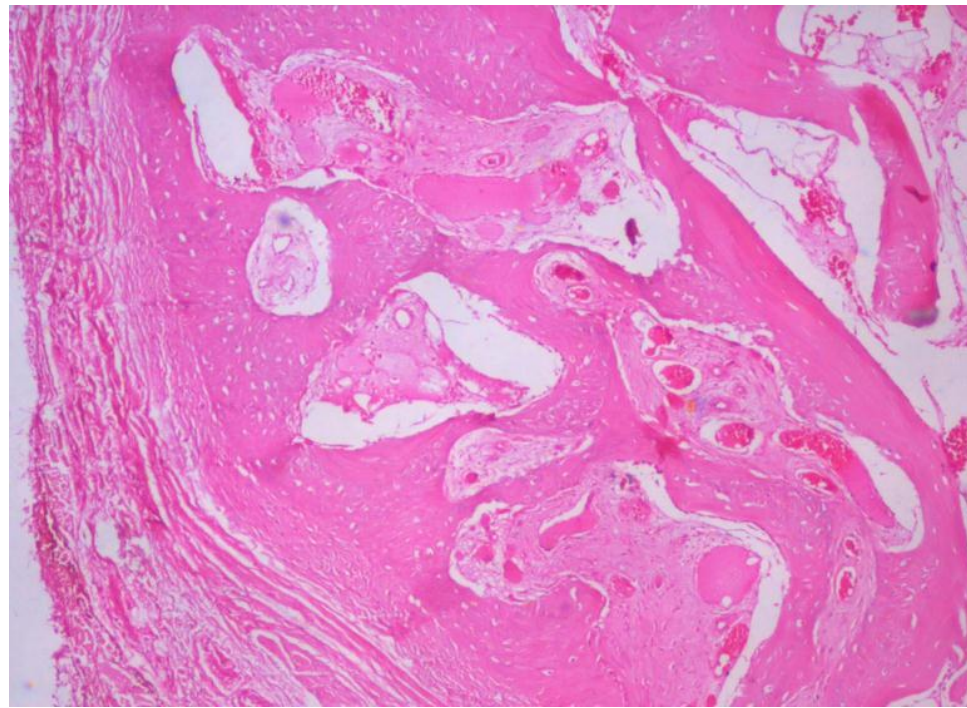

Figure 4: Photomicrograph of cancellous osteoma 